\title{
Moyamoya: An Uncommon Variant Of Stroke In Children
}

\author{
Rida Zaheer, Ayesha Ahmed, Shazia Shakoor, Shakeel Ahmed
}

\begin{abstract}
:
Moyamoya is a rare cause of stroke in children. It is an infrequent cerebrovascular disorder of unknown etiology. We are reporting a case of a 7-year-old girl who presented with an acute history of left-sided weakness. On imaging she was diagnosed with Moyamoya disease. CT Angiogram revealed an occlusion of the right middle cerebral artery typical of Moyamoya disease. The child improved with conservative therapy.
\end{abstract}

Key words: stroke, Moyamoya disease, children, transient ischemia

\section{INTRODUCTION:}

Moyamoya disease is an uncommon variant of stroke of unknown etiology in children, characterized by occlusion of the cerebral circulation at the level of the circle of Willis. Obstruction leads to multiple ischemic strokes with neurological deficit ${ }^{1}$. Occurrence in children is relatively rare i.e. six in every 100,000 children and at least one-third of these cases occur in newborns, being more common under the age of $2 \mathrm{yrs}^{2}$. Moyamoya, which in Japanese means "puff of smoke", was first reported in 1957 in Japan as an occlusion of the bilateral internal carotid arteries. The disease is diagnosed radiologically on angiography as a puffy smoke like appearance due to the formation of collaterals, hence the name Moyamoya. It is a slowly progressive disease in which there is bilateral occlusion and aneurysms of the middle cerebral and internal carotid arteries with the development of collateral circulation ${ }^{3}$. Clinical manifestations include neurological deficits like hemiparesis, monoparesis and other sensory disturbances in children ${ }^{4}$.

\section{CASE REPORT:}

A 7-year-old girl brought to the emergency department with the complaint of weakness of left side of the body for one day and inability to move the same side and dribbling from the mouth for 3-4 hrs. According to her father, the child was in good health a day back, when, in the morning she suddenly felt weakness in the left side of her body and was unable to stand or walk. Her father noticed that she was unable to move her left arm and leg, which was associated with

Rida Zaheer,
I Degistrar, Department of Paediatrics,
I Ayesha Ahmed,
I Final Year Student
| Dow University of Health Sciences, Karachi
| Shazia Shakoor,
| Professor of Physiology,
| Bahria University Medical \& Dental College, Karachi
| Shakeel Ahmed
| Professor of Paediatrics,
| Rahria University Medical And Dental College, Karachi.
| Received: 15-05-18

difficulty in speech and loss of sensation over the left side of the body. She also developed difficulty in taking food and drinking water with dribbling from the mouth. There were no complaints of a headache, fits, visual disturbance or trauma. Past medical history was uneventful and there was no previous hospitalization. The child was fully immunized according to EPI. The perinatal period was uneventful, and all milestones were achieved at appropriate ages. There was no family history of any inherited or any bleeding disorder.

Detailed neurological examination revealed a conscious child, well oriented with time, place and person, understanding and following the commands, having difficulty in speech. She had an evident left-sided facial asymmetry. She was unable to walk without support, with obvious limping on her left side. Motor and sensory examination on the right side was normal. Left upper and lower limbs were flaccid with hypotonia but the bulk was normal, power was grade 0 , and reflexes were absent. There were no involuntary movements. Cranial nerve examination revealed right supranuclear facial paralysis (left sided facial weakness) with lesion of left accessory and right hypoglossal nerve. Rest of the general physical and systemic examination was unremarkable.

Initial investigations included complete blood cell count which showed normocytic anemia with normal leukocyte and platelet counts. Coagulation studies including prothrombin time and absolute partial prothrombin time were within normal limits. Specific tests for hypercoagulability disorders included activated protein $\mathrm{C}$ resistance, anti-cardiolipin antibody, anti-thrombin III, homocysteine, D dimer, Factor V Leiden, fibrinogen, lupus anticoagulant, protein $\mathrm{S}$, and thrombin time which were all within the normal range. CT angiography of the brain shows occlusion at the proximal portion of the right middle cerebral artery. There were abnormal vascular collaterals in the vicinity of the occlusive areas suggestive of Moyamoya disease (Fig1).

The child was started on low molecular weight heparin for 2 weeks followed by oral aspirin with supportive care, and physiotherapy was also continued. The child showed significant improvement and at 3 months follow-up showed full recovery with no residual deficit. Repeat $\mathrm{CT}$ angiography 
revealed re-vascularization of the occlusive artery (Fig2).

\section{DISCUSSION:}

Moyamoya is a rare progressive Cerebro-occlusive disease

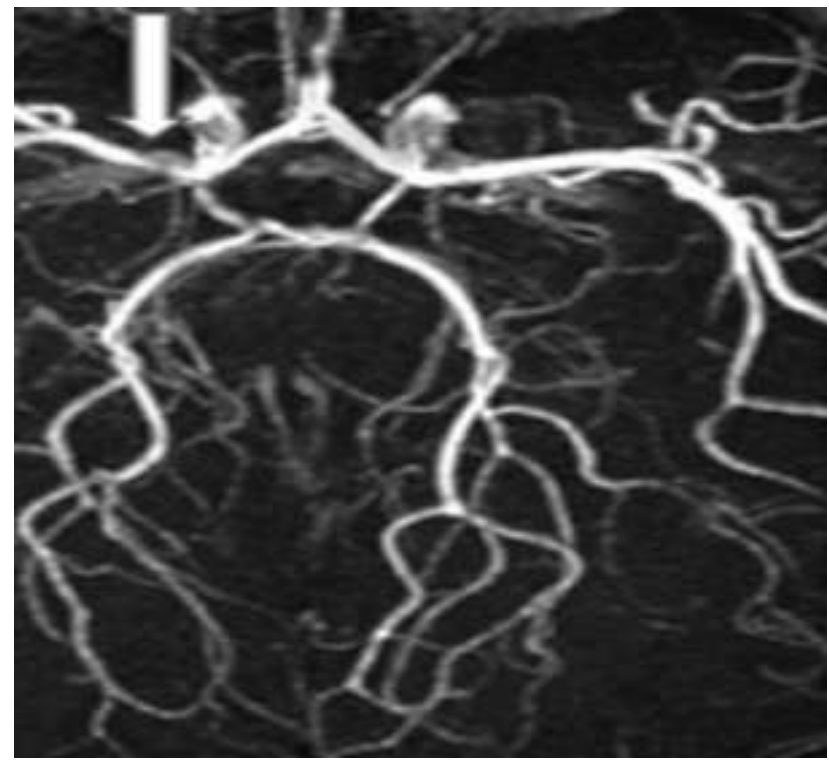

Fig 1: CT angiogram shows occlusion of the right middle cerebral artery

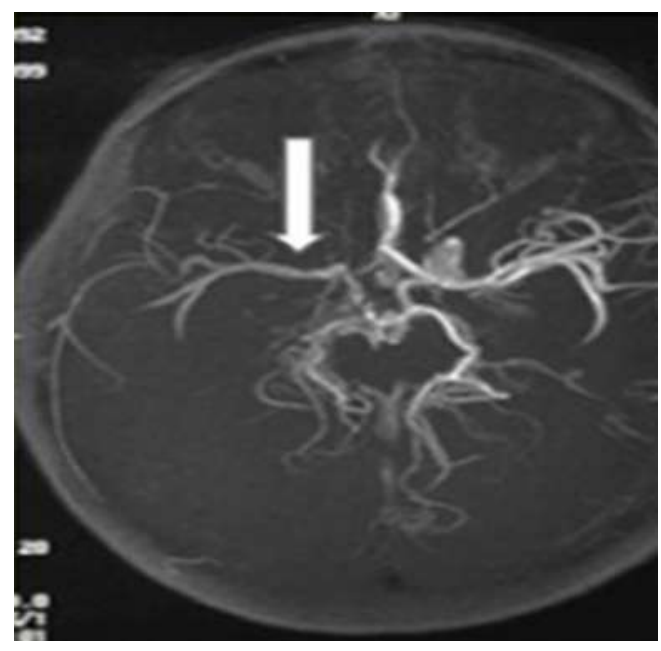

Fig 2: Repeat CT angiogram reveals reperfusion of the occlusive artery

that involves vessels of the cerebral circulation at the level of the circle of Willis ${ }^{3}$. Initially it was reported in Japan but now it is prevalent globally. The etiology is still unknown but genetic predilection is identified to play a part but it is not confirmed yet ${ }^{5}$. The symptoms of the disease vary from cerebral vascular occlusion/stenosis to hemorrhage leading to ischemic stroke, neurological deficit, and hemiplegia ${ }^{3}$. Ahmed R et al in 1997 reported four patients of this disease variant who presented with hemiparesis ${ }^{6}$. Similar cases were reported in four and five-year-old children by Rafiq A et al and Shamim $S$ et al respectively ${ }^{7,8} .13$ patients were reported by Sana et al at Aga Khan University hospital during the period of 1988 -2006; their results showed the mean age at presentation is 16 years and a female preponderance. They also concluded that only 3 patients required surgery whereas the rest were treated conservatively ${ }^{9}$. Diagnosis is established by imaging studies. CT angiography is the gold standard in the diagnosis and follow up in patients with Moyamoya disease ${ }^{10}$. Anticoagulation including heparin or warfarin is the main stay of therapy in this disease but is of unproven benefit. This therapy can be empirically considered in ischemic stroke or when thrombosis of vessels is present to prevent future ischemic strokes. Safety and efficacy for these drugs have not been fully established, and careful monitoring of risk and benefits is required. The basis for the administration of anticoagulation and antiplatelet therapy is the prevention of further strokes. These drugs do not modify the natural course of the disease rather significantly increase the risk of hemorrhage with large strokes ${ }^{11}$. Various surgical procedures have also been used in the treatment of Moyamoya disease, with the goal of re-vascularizing the ischemic hemisphere ${ }^{12}$. Rehabilitation treatment includes physical, occupational and speech therapy should be considered, depending on the neurologic impairment.

This case highlights the importance of early diagnosis and management that lead to a favorable outcome in children with Moyamoya disease. Prognosis of patients with Moyamoya disease is found to be related to age and the type of presentation. Disease has a more rapid and worse prognosis in children younger than 3 years than in those aged 3 years or older.

\section{CONCLUSION:}

Although the disease is not common, it should be well considered while dealing with stroke in children, especially during the first decade. This disease, in the future, might be helpful in understanding the pathogenesis of vaso-occlusive diseases in children.

\section{REFERENCES:}

1. Broderick J, Talbot GT, Prenger E, Leach A, Brott T. Stroke in children within a major metropolitan area: the surprising importance of intracerebral hemorrhage. J Child Neurol 1993; 8:250-5.

2. Roy Sucholeiki, Amy Kao. Moyamoya Disease Clinical Presentation: emedicine. Medscape.com/article/1180952-Jan 25, 2015.

3. Kim JS. Moyamoya Disease: Epidemiology, Clinical Features, and Diagnosis. J Stroke. 2016;18(1):2-11.

4. Gosalakkal JA. Moyamoya disease: a review Neurology India 2002;50(1):6-10.

5. Gaillard J, Klein J, Duran D. J Neurosurg Pediatr. 2017; 19(5):553-559.

6. Ahmed R, Ahsan H Imaging of Moyamoya disease. The journal of Pakistan Medical Association 1997, 47(7):181-5. 
Rida Zaheer, Ayesha Ahmed, Shazia Shakoor, Shakeel Ahmed

7. Rafiq A, Vaqar A, Javaid KH, Parveen R. Moyamoya disease. J Coll Physicians Surg Pak. 2011;21(8):506-8.

8. Shamim S, Kumar J, Jamalvi SW, . Moyamoya disease in a child. J Coll Physicians Surg Pak 2008;18(4):252-3.

8. Janda PH, Bellew JG, Veerappan V. Moyamoya disease: case report and literature review. J Am Osteopath Assoc. 2009; 109(10):547-53.

9. Sana Shoukat, Ahmed Itrat, Ather M Taqui, Moyamoya disease: A clinical spectrum, literature review and case series from a tertiary care hospital in Pakistan BMC Neurology 2009, 9:15.
10. Deng Y, Wang Y, Yang W, J Child Neurol. 2015;30(3):33943.

11. David D. Gonda, Jared Fridley, Andrew Jea, The safety and efficacy of use of low-molecular-weight heparin in pediatric neurosurgical patients. J Neurosurg Pediatr 16:329-334, 2015.

12. Fung LW, Thompson D, Ganesan V. Revascularisation surgery for paediatric moyamoya: a review of the literature. Childs Nerv Syst. 2005;21(5):358-64 